\title{
Determination of critical observed frequencies in chi square
}

\author{
L. W. BUCKALEW and W. H. PEARSON \\ Aerospace Medical Research Laboratory, Wright-Patterson Air Force Base, Dayton, Ohio 45433
}

\begin{abstract}
The chi-square goodness-of-fit test is viewed as restrictive in interpretation potentials. The concept, rationale, formulas, and limitations for a simple test of observed frequencies are offered. This test, respecting limitations, allows the determination of a critical observed frequency value for any cell that would yield a significant deviation from expectations. The test is statistically conservative and allows category- or condition-specific interpretation of chi-square data.
\end{abstract}

Chi square is a basic nonparametric statistic used in situations in which it is desirable to compare a set of observed frequencies with a set of theoretical frequencies. By virture of mathematical definition, $\chi^{2}=$ $\Sigma\left[(\mathrm{O}-\mathrm{E})^{2} / \mathrm{E}\right]$, chi square is a descriptive measure of the magnitude of the difference between the observed and expected (theoretical) frequencies (Ferguson, 1976), with the proportion of measurements falling in each category of the presumed population deduced from the null hypothesis (Siegel, 1956). The larger the computed chi square, the more difference exists between observed and expected frequencies and the less likely it is that differences can be explained by sampling fluctuation; that is, it is less likely that these differences could have occurred under $\mathrm{H}_{0}$.

There are two basic chi-square tests, goodness of fit and independence, although Ferguson (1976) argues that the latter is a particular case of the former. Of present interest, a test of goodness of fit, referred to by Siegel (1956) as the chi-square one-sample test, compares a set of observed frequencies on a single variable with a corresponding set of expected frequencies, typically conforming to a known distribution. Present interest is vested in the goodness-of-fit test wherein cell expected frequencies are equal.

The allowable conclusion for a significant chi-square value in a goodness-of-fit situation is limited to the general statement that, for a given variable, the distribution of observed frequencies is different from that of expected frequencies. Determination of reason, source, or major contributing factor for this difference is left to speculation unless every possible reduction (k-1) to a $\mathrm{df}=1$ condition is produced in which each chi-square value is interpreted as a normal deviate $\left(z=\sqrt{\chi^{2}}\right)$. While laborious and time-consuming, this operation would allow statistical designation of the cell or cells

The senior author accomplished this work while assigned to the Summer Faculty Research Progam. Requests for reprints may be addressed to L. W. Buckalew, Department of Psychology, Box 200, Alabama A \& M University, Normal, Alabama 35752. responsible for the significance of the original chi square.

The broad spectrum of categorical variables amenable to a goodness-of-fit chi-square test enhances the appropriateness of this analysis to a diverse range of applications [e.g., drug efficacy studies (psychopharmacology), evaluation of therapeutic modes (clinical psychology), scaling or opinion research (social psychology), perceptual discrimination tasks (psychophysics), product or situational analyses (industrial psychology), and other categorization/evaluation tasks]. In many judgmental situations, it is experimentally desirable to identify the category or condition that significantly contributed to the value of chi square, assuming its significance. However, exacting interpretation of chi square demands a specificity beyond the traditional scope of consideration.

The proffered formulas allow identification of the cell or cells responsible for the significance of a chi square and preclude the need to calculate chi square. These formulas require only consideration of an observed frequency $\left(\mathrm{O}_{\mathrm{f}}\right)$, given the known quantities of $\mathrm{df}$, expected frequency $\left(\mathrm{E}_{\mathrm{f}}\right)$, and critical value of chi square needed for significance at a stated level of probability $\left(\chi_{\text {crit }}^{2}\right)$.

For determining significantly high or low observed frequencies, Formulas 1 and 2, respectively, apply:

$$
\begin{aligned}
& O_{f_{\text {crit }}} \geqslant E_{f}+\sqrt{\left(E_{f}\right)\left(\chi^{2} \text { crit }\right)} \\
& O_{\text {fcrit }} \leqslant E_{f}-\sqrt{\left(E_{f}\right)\left(\chi^{2} \text { crit }\right)}
\end{aligned}
$$

Determination of a critical $\mathrm{O}_{\mathrm{f}}$ value must respect the following limitations: (1) $\mathrm{df}>1$, (2) $\mathrm{E}_{\mathrm{f}} \geqslant \chi^{2}$ crit, and (3) equal cell expected frequencies (i.e., $E_{f}=N / k$ ). Limitation 2 is a necessary consideration only when interest is in observed frequencies lower than expected.

Given realization of the high or low critical $\mathrm{O}_{f}$, the significance of a traditionally calculated chi square is assured and the cell or cells (category/condition) responsible for chi-square significance is immediately identified. Ultimately, interpretation of chi square becomes 
appreciably more specific as to the major contributing factor to significance. Obviously, if the critical value of an $\mathrm{O}_{\mathrm{f}}$ is reached, the now unnecessary but traditional computation of chi square will yield a significant value. In this respect, the critical $O_{f}$ test is very conservative: No critical $\mathrm{O}_{\mathrm{f}}$ can be identified in the absence of a significant chi square, and the existence of a significant chi square does not guarantee that a critical $O_{f}$ exists, as chi-square components are cumulative.

The mathematical proof of this conceptualization of a critical $O_{f}$ is as follows: From Formula $1, O_{f} \geqslant$ $\sqrt{E_{\mathrm{f}}}\left(\sqrt{E_{\mathrm{f}}}+\sqrt{\chi_{\text {crit }}^{2}}\right), \quad O_{\mathrm{f}}\left(\sqrt{\mathrm{E}_{\mathrm{f}}}-\sqrt{\chi^{2} \text { crit }}\right) \geqslant \sqrt{E_{\mathrm{f}}}\left(\mathrm{E}_{\mathrm{f}}-\right.$ $\chi^{2}$ crit); for $\mathrm{O}_{\mathrm{f}}>0, \mathrm{E}_{\mathrm{f}}>\chi^{2}$ crit. From Formula 2, $\mathrm{O}_{\mathrm{f}} \leqslant \sqrt{E_{\mathrm{f}}}\left(\sqrt{E_{\mathrm{f}}}-\sqrt{\chi^{2} \text { crit }}\right) ;$ for $\mathrm{O}_{\mathrm{f}}>0, \mathrm{E}_{\mathrm{f}}>\chi^{2}$ crit.
These formulas are predicated on the logic that, if a single cell produces an $(\mathrm{O}-\mathrm{E})^{2} / \mathrm{E}$ value that equals or exceeds the critical value of chi square, the ultimate computed chi square will be significant and that cell may be designated as a significant contributor.

\section{REFERENCES}

Ferguson, G. A. Statistical analysis in psychology and education (4th ed.). New York: McGraw-Hill, 1976.

Siegel, S. Nonparametric statistics for the behavioral sciences. New York: McGraw-Hill, 1956.

(Received for publication September 21, 1981.) 